\title{
A particle swarm pattern search method for bound constrained global optimization
}

\author{
A. Ismael F. Vaz • Luís N. Vicente
}

Received: 12 February 2006 / Accepted: 3 January 2007 / Published online: 6 February 2007

(C) Springer Science+Business Media B.V. 2007

\begin{abstract}
In this paper we develop, analyze, and test a new algorithm for the global minimization of a function subject to simple bounds without the use of derivatives. The underlying algorithm is a pattern search method, more specifically a coordinate search method, which guarantees convergence to stationary points from arbitrary starting points. In the optional search phase of pattern search we apply a particle swarm scheme to globally explore the possible nonconvexity of the objective function. Our extensive numerical experiments showed that the resulting algorithm is highly competitive with other global optimization methods also based on function values.
\end{abstract}

Keywords Direct search · Pattern search · Particle swarm - Derivative free optimization · Global optimization · Bound constrained nonlinear optimization

AMS subject classifications $\quad 90 \mathrm{C} 26 \cdot 90 \mathrm{C} 30 \cdot 90 \mathrm{C} 56$

Support for A. Ismael F. Vaz was provided by Algoritmi Research Center, and by FCT under grants POCI/MAT/59442/2004 and POCI/MAT/58957/2004.

Support for Luís N. Vicente was provided by Centro de Matemática da Universidade de Coimbra and by FCT under grant POCI/MAT/59442/2004.

A. I. F. Vaz $(\bowtie)$

Departamento de Produção e Sistemas, Escola de Engenharia,

Universidade do Minho,

Campus de Gualtar, 4710-057 Braga, Portugal

e-mail: aivaz@dps.uminho.pt

L. N. Vicente

Departamento de Matemática,

Universidade de Coimbra,

3001-454 Coimbra, Portugal

e-mail: lnv@mat.uc.pt 


\section{Introduction}

Pattern and direct search methods are one of the most popular classes of methods to minimize functions without the use of derivatives or of approximations to derivatives [26]. They are based on generating search directions which positively span the search space. Direct search is conceptually simple and natural for parallelization. These methods can be designed to rigorously identify points satisfying stationarity for local minimization (from arbitrary starting points). Moreover, their flexibility can be used to incorporate algorithms or heuristics for global optimization, in a way that the resulting direct or pattern search method inherits some of the properties of the imported global optimization technique, without jeopardizing the convergence for local stationarity mentioned before.

The particle swarm optimization algorithm was firstly proposed in ref. [12,24] and has received some recent attention in the global optimization community $[7,35]$. The particle swarm algorithm tries to simulate the social behavior of a population of agents or particles, in an attempt to optimally explore some given problem space. At a time instant (an iteration in the optimization context), each particle is associated with a stochastic velocity vector which indicates where the particle is moving to. The velocity vector for a given particle at a given time is a linear stochastic combination of the velocity in the previous time instant, of the direction to the particle's best position, and of the direction to the best swarm positions (for all particles). The particle swarm algorithm is a stochastic algorithm in the sense that it relies on parameters drawn from random variables, and thus different runs for the same starting swarm may produce different outputs. Some of its advantages are being simple to implement and easy to parallelize. It depends, however, on a few of parameters which influence the rate of convergence in the vicinity of the global optimum. Overall it does not require many user-defined parameters, which is important for practitioners that are not familiar with optimization. Some numerical evidence seems to show that particle swarm can outperform genetic algorithms on difficult problem classes, namely for unconstrained global optimization problems [6]. Moreover, it fits nicely into the pattern search framework.

The goal of this paper is to show how particle swarm can be incorporated in the pattern search framework. The resulting particle swarm pattern search algorithm is still a pattern search algorithm, producing sequences of iterates along the traditional requirements for this class of methods (based on integer lattices and positive spanning sets). The new algorithm is better equipped for global optimization because it is more aggressive in the exploration of the search space. Our numerical experiences showed that a large percentage of the computational work is spent in the particle swarm component of pattern search.

Within the pattern search framework, the use of the search step for surrogate optimization [5,30] or global optimization [1] is an active area of research. Hart has also used evolutionary programming to design evolutionary pattern search methods (see [16] and the references therein). There are some significative differences between his work and ours. First, we are exclusively focused on global optimization and our heuristic is based on particle swarm rather than on evolutionary algorithms. Further, our algorithm is deterministic in its pattern search component. As a result, we obtain that a subsequence of the mesh size parameters tends to zero in the deterministic sense rather than with probability one like in Hart's algorithms. 
We are interested in solving optimization problems of the form

$$
\min _{z \in \mathbb{R}^{n}} f(z) \quad \text { s.t. } \quad z \in \Omega
$$

with

$$
\Omega=\left\{z \in \mathbb{R}^{n}: \ell \leq z \leq u\right\},
$$

where the inequalities $\ell \leq z \leq u$ are posed componentwise and $\ell \in(-\infty, \mathbb{R})^{n}$, $u \in(\mathbb{R},+\infty)^{n}$, and $\ell<u$. There is no need to assume any type on smoothness on the objective function $f(z)$ to apply particle swarm or pattern search. To study the convergence properties of pattern search, and thus of the particle swarm pattern search method, one has to impose some smoothness on $f(z)$, in particular to characterize stationarity at local minimizers.

The next two sections are used to describe the particle swarm paradigm and the basic pattern search framework. We introduce the particle swarm pattern search method in Sect. 4. The convergence and termination properties of the proposed method are discussed in Sect. 5. A brief review about the optimization solvers used in the numerical comparisons and implementation details about our method are given in Sect. 6. The numerical results are presented in Sect. 7 for a large set of problems. We end the paper in Sect. 8 with conclusions and directions for future work.

\section{Particle swarm}

In this section, we briefly describe the particle swarm optimization algorithm. Our description follows the presentation of the algorithm tested in ref. [6] and the reader is pointed to ref. [6] for other algorithmic variants and details.

The particle swarm optimization algorithm is based on a population (swarm) of $s$ particles, where $s$ is known as the population size. Each particle is associated with a velocity which indicates where the particle is moving to. Let $t$ be a time instant. The new position $x^{i}(t+1)$ of the $i$ th particle at time $t+1$ is computed by adding to the old position $x^{i}(t)$ at time $t$ a velocity vector $v^{i}(t+1)$ :

$$
x^{i}(t+1)=x^{i}(t)+v^{i}(t+1)
$$

for $i=1, \ldots, s$.

The velocity vector associated to each particle $i$ is updated by

$$
v_{j}^{i}(t+1)=\iota(t) v_{j}^{i}(t)+\mu \omega_{1 j}(t)\left(y_{j}^{i}(t)-x_{j}^{i}(t)\right)+v \omega_{2 j}(t)\left(\hat{y}_{j}(t)-x_{j}^{i}(t)\right)
$$

for $j=1, \ldots, n$, where $\iota(t)$ is a weighting factor (called inertial) and $\mu$ and $\nu$ are positive real parameters (called, in the particle swarm terminology, the cognition parameter and the social parameter, respectively). The numbers $\omega_{1 j}(t)$ and $\omega_{2 j}(t), j=1, \ldots, n$, are randomly drawn from the uniform $(0,1)$ distribution. Finally, $y^{i}(t)$ is the position of the $i$ th particle with the best objective function value so far calculated, and $\hat{y}(t)$ is the particle position with the best (among all particles) objective function value found so far. The update formula (2) adds to the previous velocity vector a stochastic combination of the directions to the best position of the $i$ th particle and to the best (among all) particles position.

The position $\hat{y}(t)$ can be described as

$$
\hat{y}(t) \in \operatorname{argmin}_{z \in\left\{y^{1}(t), \ldots, y^{s}(t)\right\}} f(z) .
$$


The argmin operator can return a set. When that happens in this situation, it is the first element in this argmin set that matches the implementations, since the best element is only updated algorithmically when a new one is found yielding a decrease in the objective function.

The bound constraints in the variables are enforced by considering the projection onto $\Omega$, given for all particles $i=1, \ldots, s$ by

$$
\operatorname{proj}_{\Omega}\left(x_{j}^{i}(t)\right)= \begin{cases}\ell_{j}, & \text { if } x_{j}^{i}(t)<\ell_{j}, \\ u_{j}, & \text { if } x_{j}^{i}(t)>u_{j}, \\ x_{j}^{i}(t), & \text { otherwise }\end{cases}
$$

for $j=1, \ldots, n$. This projection must be applied to the new particles positions computed by Eq. (1).

The stopping criterion of the algorithm should be practical and has to ensure proper termination. One possibility is to stop when the norm of the velocities vector is small for all particles. It is possible to prove under some assumptions and for some algorithmic parameters that the expected value of the norm of the velocities vectors tends to zero for all particles (see also the analysis presented in Sect. 5).

The particle swarm optimization algorithm is described in Algorithm 2.1.

\section{Algorithm 2.1}

1. Choose a population size $s$ and a stopping tolerance $v_{\text {tol }}>0$. Randomly initialize the initial swarm positions $x^{1}(0), \ldots, x^{s}(0)$ and the initial swarm velocities $v^{1}(0), \ldots, v^{s}(0)$.

2. Set $y^{i}(0)=x^{i}(0), i=1, \ldots, s$, and $\hat{y}(0) \in \arg \min _{z \in\left\{y^{1}(0), \ldots, y^{s}(0)\right\}} f(z)$. Let $t=0$.

3. Set $\hat{y}(t+1)=\hat{y}(t)$.

For $i=1, \ldots, s$ do (for every particle $i$ ):

- Compute $\hat{x}^{i}(t)=\operatorname{proj}_{\Omega}\left(x^{i}(t)\right)$.

- If $f\left(\hat{x}^{i}(t)\right)<f\left(y^{i}(t)\right)$ then

(a) Set $y^{i}(t+1)=\hat{x}^{i}(t)$ (update the particle $i$ best position).

(b) If $f\left(y^{i}(t+1)\right)<f(\hat{y}(t+1))$ then $\hat{y}(t+1)=y^{i}(t+1)$ (update the particles best position).

- Otherwise set $y^{i}(t+1)=y^{i}(t)$.

4. Compute $v^{i}(t+1)$ and $x^{i}(t+1), i=1, \ldots, s$, using formulae (1) and (2).

5. If $\left\|v^{i}(t+1)\right\|<v_{\text {tol }}$, for all $i=1, \ldots, s$, then stop. Otherwise, increment $t$ by one and go to Step 3 .

\section{Pattern search}

Direct search methods are an important class of optimization algorithms which attempt to minimize a function by comparing, at each iteration, its value in a finite set of trial points (computed by simple mechanisms). Direct search methods not only do not use any derivative information but also do not try to implicitly build any type of derivative approximation. Pattern search methods can be seen as direct search methods for which the rules of generating the trial points follow stricter calculations and for which convergence for stationary points can be proved from arbitrary starting points. A comprehensive review of direct and pattern search can be found in ref. [26], where a broader class of methods referred to as 'generating set search' is described. 
In this paper, we prefer to describe pattern search methods using the search/poll step framework [3], since it better suits the incorporation of heuristic procedures.

The central notion in pattern search are positive spanning sets. The definitions and properties of positive spanning sets and of positive bases are given, for instance, in ref. $[10,26]$. One of the simplest positive spanning sets is formed by the vectors of the canonical basis and their negatives:

$$
D_{\oplus}=\left\{e_{1}, \ldots, e_{n},-e_{1}, \ldots,-e_{n}\right\} .
$$

The set $D_{\oplus}$ is also a (maximal) positive basis. The elementary direct search method based on this positive spanning set is known as coordinate or compass search and its structure is basically all we need in this paper.

Given a positive spanning set $D$ and the current iterate ${ }^{1} y(t)$, we define two sets of points: the mesh $M_{t}$ and the poll set $P_{t}$. The mesh $M_{t}$ is given by

$$
M_{t}=\left\{y(t)+\alpha(t) D z, z \in \mathbb{Z}_{+}^{|D|}\right\},
$$

where $\alpha(t)>0$ is the mesh size parameter (also known as the step-length control parameter) and $\mathbb{Z}_{+}$is the set of nonnegative integers. The mesh has to meet some integrality requirements for the method to achieve global convergence to stationary points, in other words, convergence to stationary points from arbitrary starting points. In particular, the matrix $D$ has to be of the form $G \hat{Z}$, where $G \in \mathbb{R}^{n \times n}$ is a nonsingular generating matrix and $\hat{Z} \in \mathbb{Z}^{n \times|D|}$. The positive basis $D_{\oplus}$ satisfies this requirement trivially when $G$ is the identity matrix.

The search step conducts a finite search in the mesh $M_{t}$. The poll step is executed only if the search step fails to find a point for which $f$ is lower than $f(y(t))$. The poll step evaluates the function at the points in the poll set

$$
P_{t}=\{y(t)+\alpha(t) d, d \in D\}
$$

trying to find a point where $f$ is lower than $f(y(t))$. Note that $P_{t}$ is a subset of $M_{t}$. If $f$ is continuously differentiable at $y(t)$, the poll step is guaranteed to succeed if $\alpha(t)$ is sufficiently small, since the positive spanning set $D$ contains at least one direction of descent (which makes an acute angle with $-\nabla f(y(t))$ ). Thus, if the poll step fails then the mesh size parameter must be reduced. It is the poll step that guarantees the global convergence of the pattern search method.

In order to generalize pattern search for bound constrained problems it is necessary to use a feasible initial guess $y(0) \in \Omega$ and to keep feasibility of the iterates by rejecting any trial point, that is, out of the feasible region. Rejecting infeasible trial points can be accomplished by applying a pattern search algorithm to the following penalty function

$$
\hat{f}(z)= \begin{cases}f(z), & \text { if } z \in \Omega \\ +\infty, & \text { otherwise. }\end{cases}
$$

The iterates produced by a pattern search method applied to the unconstrained problem of minimizing $\hat{f}(z)$ coincide trivially with those generated by the same type of pattern search method, but applied to the minimization of $f(z)$ subject to simple bounds and to the rejection of infeasible trial points.

1 We will use $y(t)$ to denote the current iterate, rather than $x_{k}$ or $y_{k}$, to follow the notation of the particle swarm framework. 
It is also necessary to include in the search directions $D$ those directions that guarantee the presence of a feasible descent direction at any nonstationary point of the bound constrained problem. One can achieve this goal in several ways. But, since $D_{\oplus}$ includes all such directions [26], we assume the use of this set throughout the remainder of the paper.

In order to completely describe the basic pattern search algorithm, we need to specify how to increase and decrease the mesh size or step-length control parameter $\alpha(t)$. These expansions and contractions use the factors $\phi(t)$ and $\theta(t)$, respectively, which must obey to the following rules:

$$
\begin{array}{lll}
\phi(t)=\bar{\tau}^{\ell_{t}}, & \text { for some } \ell_{t} \in\left\{0, \ldots, \ell_{\max }\right\}, & \text { if } t \text { is successful, } \\
\theta(t)=\bar{\tau}^{m_{t}}, & \text { for some } m_{t} \in\left\{m_{\min }, \ldots,-1\right\}, & \text { if } t \text { is unsuccessful, }
\end{array}
$$

where $\bar{\tau}>1$ is a positive rational, $\ell_{\max }$ is a nonnegative integer, and $m_{\min }$ is a negative integer, chosen at the beginning of the method and unchanged with $t$. For instance, we can have $\theta(t)=1 / 2$ for unsuccessful iterations and $\phi(t)=1$ or $\phi(t)=2$ for successful iterations.

The basic pattern search method for use in this paper is described in Algorithm 3.1.

\section{Algorithm 3.1}

1. Choose a positive rational $\bar{\tau}$ and the stopping tolerance $\alpha_{\text {tol }}>0$. Choose the positive spanning set $D=D_{\oplus}$.

2. Let $t=0$. Select an initial feasible guess $y(0)$. Choose $\alpha(0)>0$.

3. [Search Step]

Evaluate $f$ at a finite number of points in $M_{t}$. If a point $z(t) \in M_{t}$ is found for which $\hat{f}(z(t))<\hat{f}(y(t))$ then set $y(t+1)=z(t), \alpha(t+1)=\phi(t) \alpha(t)$ (optionally increasing the mesh size parameter), and declare successful both the search step and the current iteration.

\section{4. [Poll Step]}

Skip the poll step if the search step was successful.

- If there exists $d(t) \in D$ such that $\hat{f}(y(t)+\alpha(t) d(t))<\hat{f}(y(t))$ then

(a) Set $y(t+1)=y(t)+\alpha(t) d(t)$ (poll step and iteration successful).

(b) Set $\alpha(t+1)=\phi(t) \alpha(t)$ (optionally increase the mesh size parameter).

- Otherwise, $\hat{f}(y(t)+\alpha(t) d(t)) \geq \hat{f}(y(t))$ for all $d(t) \in D$, and

(a) Set $y(t+1)=y(t)$ (iteration and poll step unsuccessful).

(b) Set $\alpha(t+1)=\theta(t) \alpha(t)$ (reduce the mesh size parameter).

5. If $\alpha(t+1)<\alpha_{\text {tol }}$ then stop. Otherwise, increment $t$ by one and go to Step 3 .

An example of the use of the search step is given in the next section. The poll step can be implemented in a number of different ways. The polling can be opportunistic (when it quits once the first decrease in the objective function is found) or complete (when the objective function is evaluated at all the points of the poll set). The order in which the points in $P_{t}$ are evaluated can also differ [4,9].

\section{The particle swarm pattern search method}

Pattern search methods are local methods in the sense that they are designed to achieve convergence (from arbitrary starting points) to points that satisfy necessary conditions for local optimality. Some numerical experience has shown cases in which 
pattern search has found global minimizers for certain classes of problems (see, for instance, $[1,31]$ ). Certain parameter choices can enable pattern search to jump out of one basin of attraction of a local minimizer into another (that is hopefully a better one). This paper is an attempt to exploit this tendency by applying a global heuristic in the search step. On the other hand, the poll step can rigorously guarantee convergence to stationary points.

The hybrid method introduced in this paper is a pattern search method that incorporates a particle swarm search in the search step. The idea is to start with an initial population and to apply one step of particle swarm at each search step. Consecutive iterations where the search steps succeed reduce to consecutive iterations of particle swarm, in an attempt to identify a neighborhood of a global minimizer. Whenever the search step fails, the poll step is applied to the best position over all particles, performing a local search in the poll set centered at this point.

The points calculated in the search step by the particle swarm scheme must belong to the pattern search mesh $M_{t}$. This task can be done in several ways and, in particular, one can compute their 'projection' onto $M_{t}$

$$
\operatorname{proj}_{M_{t}}\left(x^{i}(t)\right)=\min _{u \in M_{t}}\left\|u-x^{i}(t)\right\|
$$

for $i=1, \ldots, s$, or an approximation thereof.

There is no need then to project onto $\Omega$ since the use of the penalty function $\hat{f}$ in pattern search takes care of the bound constraints.

The stopping criterion of the particle swarm pattern search method is the conjunction of the stopping criteria for particle swarm and pattern search. The particle swarm pattern search method is described in Algorithm 4.1.

\section{Algorithm 4.1}

1. Choose a positive rational $\bar{\tau}$ and the stopping tolerance $\alpha_{\text {tol }}>0$. Choose the positive spanning set $D=D_{\oplus}$.

Choose a population size $s$ and a stopping tolerance $v_{\text {tol }}>0$. Randomly initialize the initial swarm positions $x^{1}(0), \ldots, x^{s}(0)$ and the initial swarm velocities $v^{1}(0), \ldots, v^{s}(0)$.

2. Set $y^{i}(0)=x^{i}(0), i=1, \ldots, s$, and $\hat{y}(0) \in \arg \min _{z \in\left\{y^{1}(0), \ldots, y^{s}(0)\right\}} f(z)$. Choose $\alpha(0)>$ 0 . Let $t=0$.

3. [Search Step]

Set $\hat{y}(t+1)=\hat{y}(t)$.

For $i=1, \ldots, s$ do (for every particle $i$ ):

- Compute $\hat{x}^{i}(t)=\operatorname{proj}_{M_{t}}\left(x^{i}(t)\right)$.

- If $\hat{f}\left(\hat{x}^{i}(t)\right)<\hat{f}\left(y^{i}(t)\right)$ then

(a) Set $y^{i}(t+1)=\hat{x}^{i}(t)$ (update the particle $i$ best position).

(b) If $f\left(y^{i}(t+1)\right)<f(\hat{y}(t+1))$ then

* Set $\hat{y}(t+1)=y^{i}(t+1)$ (update the particles best position; search step and iteration successful).

* Set $\alpha(t+1)=\phi(t) \alpha(t)$ (optionally increase the mesh size parameter).

- Otherwise set $y^{i}(t+1)=y^{i}(t)$.

\section{4. [Poll Step]}

Skip the poll step if the search step was successful.

- If there exists $d(t) \in D$ such that $\hat{f}(\hat{y}(t)+\alpha(t) d(t))<\hat{f}(\hat{y}(t))$ then 
(a) Set $\hat{y}(t+1)=\hat{y}(t)+\alpha(t) d(t)$ (update the leader particle position; poll step and iteration successful).

(b) Set $\alpha(t+1)=\phi(t) \alpha(t)$ (optionally increase the mesh size parameter).

- Otherwise, $\hat{f}(\hat{y}(t)+\alpha(t) d(t)) \geq \hat{f}(\hat{y}(t))$ for all $d(t) \in D$, and

(a) Set $\hat{y}(t+1)=\hat{y}(t)$ (no change in the leader particle position; poll step and iteration unsuccessful).

(b) Set $\alpha(t+1)=\theta(t) \alpha(t)$ (reduce the mesh size parameter).

5. Compute $v^{i}(t+1)$ and $x^{i}(t+1), i=1, \ldots, s$, using formulae (1) and (2).

6. If $\alpha(t+1)<\alpha_{\mathrm{tol}}$ and $\left\|v^{i}(t+1)\right\|<v_{\mathrm{tol}}$, for all $i=1, \ldots, s$, then stop. Otherwise, increment $t$ by one and go to Step 3 .

\section{Convergence}

The convergence analysis studies properties of a sequence of iterates generated by Algorithm 4.1. For this purpose, we consider $\alpha_{\text {tol }}=0$ and $v_{\text {tol }}=0$, so that the algorithm never meets the termination criterion. Let $\{\hat{y}(t)\}$ be the sequence of iterates produced by Algorithm 4.1. Since all necessary pattern search ingredients are present, this method generates, under the appropriate assumptions, a sequence of iterates converging (independently of the starting point) to first-order critical points. A standard result for this class of methods tells us that there is a subsequence of unsuccessful iterations converging to a limit point and for which the mesh size parameter tends to zero [3,26].

Theorem 5.1 Let $L(\hat{y}(0))=\left\{z \in \mathbb{R}^{n}: f(z) \leq f(\hat{y}(0))\right\}$ be a bounded set. Then, there exists a subsequence $\left\{\hat{y}\left(t_{k}\right)\right\}$ of the iterates produced by Algorithm 4.1 (with $\alpha_{\mathrm{tol}}=v_{\mathrm{tol}}=$ 0) such that

$$
\lim _{k \longrightarrow+\infty} \hat{y}\left(t_{k}\right)=\hat{y}_{*} \quad \text { and } \quad \lim _{k \longrightarrow+\infty} \alpha\left(t_{k}\right)=0
$$

for some $\hat{y}_{*} \in \Omega$ and such that the subsequence $\left\{t_{k}\right\}$ consists of unsuccessful iterations.

The integrality assumptions imposed in the construction of the meshes $M_{t}$ and on the update of the mesh size parameter are fundamental for the integer lattice type arguments required to prove this result. There are other ways to obtain such a result that circumvent the need for these integrality assumptions [26], such as the imposition of a sufficient decrease condition on the step acceptance mechanism.

Depending on the differentiability properties of the objective function, different types of stationarity can be proved for the point $\hat{y}_{*}$. For instance, if the function is strictly differentiable at this point, one can prove from the positive spanning properties of $D$ that $\nabla f\left(\hat{y}_{*}\right)=0$ (see [3]). A result of the type $\liminf _{t \rightarrow+\infty}\|\nabla f(\hat{y}(t))\|=0$ can only be guaranteed when $f$ is continuously differentiable in $L(\hat{y}(0)$ ) (see [26]).

Theorem 5.1 tells us that a stopping criterion based solely on the size of the mesh size parameter (of the form $\alpha(t)<\alpha_{\text {tol }}$ ) will guarantee termination of the algorithm in a finite number of iterations. However, the stopping condition of Algorithm 4.1 also requires $\left\|v^{i}(t)\right\|<v_{\text {tol }}, i=1, \ldots, s$ (in an attempt to impose to particle swarm a desirable level of global optimization effort). Thus, it must be investigated whether or not the velocities in the particle swarm scheme satisfy a limit of the form

$$
\lim _{k \longrightarrow+\infty}\left\|v^{i}\left(t_{k}\right)\right\|=0, \quad i=1, \ldots, s .
$$


To do this we have to investigate the asymptotic behavior of the search step which is where the particle swarm strategy is applied. Rigorously speaking such a limit can only occur with probability one. To carry on the analysis we need to assume that $x^{i}(t)$, $y^{i}(t), v^{i}(t)$, and $\hat{y}(t)$ are random variables of stochastic processes. Let $E(\cdot)$ denote the appropriate mean or expected value operator.

Theorem 5.2 Suppose that for $t$ sufficiently large one has that $\iota(t), E\left(y^{i}(t)\right), i=1, \ldots, s$, and $E(\hat{y}(t))$ are constant and that $E\left(\operatorname{proj}_{M_{t}}\left(x^{i}(t-1)+v^{i}(t)\right)\right)=E\left(x^{i}(t-1)+v^{i}(t)\right)$, $i=1, \ldots, s$. Then, if the control parameters for particle swarm, $\bar{\imath}, \bar{\omega}_{1}, \bar{\omega}_{2}, \mu$, and $v$, are chosen so that $\max \{|a|,|b|\}<1$, where $\bar{\omega}_{1}=E\left(\omega_{1}(t)\right), \bar{\omega}_{2}=E\left(\omega_{2}(t)\right), \bar{\imath}=\iota(t)$ for all $t$, and $a$ and $b$ are defined, respectively, by (8) and (9), then

$$
\lim _{t \longrightarrow+\infty} E\left(v_{j}^{i}(t)\right)=0, \quad i=1, \ldots, s, j=1, \ldots, n
$$

and Algorithm 4.1 will stop almost surely in a finite number of iterations.

Proof Given that there exists a subsequence driving the mesh size parameter to zero, it remains to investigate under what conditions do the velocities tend to zero.

Consider the velocity Eq. (2), repeated here for convenience, with the indices $i$ for the particles and $j$ for the vector components dropped for simplicity. To shorten notation we write the indices $t$ as subscripts. Since $\omega_{1}(t)$ and $\omega_{2}(t)$ depend only on $t$, we get from (2) that

$$
E\left(v_{t+1}\right)=\bar{\imath} E\left(v_{t}\right)+\mu \bar{\omega}_{1}\left(E\left(y_{t}\right)-E\left(x_{t}\right)\right)+v \bar{\omega}_{2}\left(E\left(\hat{y}_{t}\right)-E\left(x_{t}\right)\right),
$$

where $\bar{\omega}_{1}=E\left(\omega_{1}(t)\right)$ and $\bar{\omega}_{2}=E\left(\omega_{2}(t)\right)$. From (2), we obtain for $v(t)$ that

$$
\begin{aligned}
E\left(v_{t}\right)= & \bar{\iota} E\left(v_{t-1}\right)+\mu \bar{\omega}_{1}\left(E\left(y_{t-1}\right)-E\left(x_{t-1}\right)\right) \\
& +v \bar{\omega}_{2}\left(E\left(\hat{y}_{t-1}\right)-E\left(x_{t-1}\right)\right) .
\end{aligned}
$$

Subtracting (5) from (4) yields

$$
\begin{aligned}
E\left(v_{t+1}\right)-E\left(v_{t}\right)= & \bar{\imath}\left(E\left(v_{t}\right)-E\left(v_{t-1}\right)\right)-\left(\mu \bar{\omega}_{1}+v \bar{\omega}_{2}\right)\left(E\left(x_{t}\right)-E\left(x_{t-1}\right)\right) \\
& +\mu \bar{\omega}_{1}\left(E\left(y_{t}\right)-E\left(y_{t-1}\right)\right)+v \bar{\omega}_{2}\left(E\left(\hat{y}_{t}\right)-E\left(\hat{y}_{t-1}\right)\right) .
\end{aligned}
$$

Noting that $x_{t}=\operatorname{proj}_{M_{t}}\left(x_{t-1}+v_{t}\right)$ we obtain the following inhomogeneous recurrence relation

$$
E\left(v_{t+1}\right)-\left(1+\bar{\imath}-\mu \bar{\omega}_{1}-v \bar{\omega}_{2}\right) E\left(v_{t}\right)+\bar{\imath} E\left(v_{t-1}\right)=g_{t},
$$

where

$$
\begin{aligned}
g_{t}= & \mu \bar{\omega}_{1}\left(E\left(y_{t}\right)-E\left(y_{t-1}\right)\right)+v \bar{\omega}_{2}\left(E\left(\hat{y}_{t}\right)-E\left(\hat{y}_{t-1}\right)\right) \\
& +\left(\mu \bar{\omega}_{1}+v \bar{\omega}_{2}\right) E\left(\operatorname{proj}_{M_{t}}\left(x_{t-1}+v_{t}\right)-\left(x_{t-1}+v_{t}\right)\right) .
\end{aligned}
$$

From the assumptions of the theorem, we have, for sufficiently large $t$, that $g_{t}$ is zero and therefore that the recurrence relation is homogeneous, with characteristic polynomial given by

$$
t^{2}-\left(1+\bar{\imath}-\mu \bar{\omega}_{1}-v \bar{\omega}_{2}\right) t+\bar{\imath}=0 .
$$

A solution of (6) is then of the form

$$
E\left(v_{t+1}\right)=c_{1} a^{t}+c_{2} b^{t}
$$


where $c_{1}$ and $c_{2}$ are constants and $a$ and $b$ are the two roots of the characteristic polynomial (7), given by

$$
\begin{aligned}
a & =\frac{\left(1+\bar{\imath}-\mu \bar{\omega}_{1}-v \bar{\omega}_{2}\right)+\sqrt{\left(1+\bar{\imath}-\mu \bar{\omega}_{1}-v \bar{\omega}_{2}\right)^{2}-4 \bar{\imath}}}{2}, \\
b & =\frac{\left(1+\bar{\imath}-\mu \bar{\omega}_{1}-v \bar{\omega}_{2}\right)-\sqrt{\left(1+\bar{\imath}-\mu \bar{\omega}_{1}-v \bar{\omega}_{2}\right)^{2}-4 \bar{\imath}}}{2} .
\end{aligned}
$$

Thus, as long as $\max \{|a|,|b|\}<1$ (which is achievable for certain choices of the control parameters $\bar{\imath}, \bar{\omega}_{1}, \bar{\omega}_{2}, \mu$, and $\left.\nu\right)$, we will get $E\left(v_{t+1}\right) \rightarrow 0$ when $t \rightarrow \infty$.

For instance, when $\bar{\omega}_{1}=\bar{\omega}_{2}=0.5$ and $\mu=v=0.5$, we obtain

$$
a=\frac{(\bar{\imath}+0.5)+\sqrt{(\bar{\imath}+0.5)^{2}-4 \bar{\iota}}}{2}
$$

and

$$
b=\frac{(\bar{\imath}+0.5)-\sqrt{(\bar{\imath}+0.5)^{2}-4 \bar{\iota}}}{2} .
$$

In this case, $\max \{|a|,|b|\}<1$ for any $0<\bar{\imath}<1$. One can clearly observe this fact in Fig. 1. Zooming into the picture reveals that for $\bar{\imath}<0.0858$ we have $(\bar{\imath}+0.5)^{2}-4 \bar{\iota} \geq 0$, resulting in two real roots for the characteristic polynomial. For $\bar{\imath} \geq 0.0858$ we have two complex conjugate roots whose moduli are equal.

It is difficult to show that the conditions of Theorem 5.2 can be rigorously satisfied, but it can be given some indication of their practical reasonability.

Given that the function is bounded below in $L(y(0))$, it is known that the monotonically decreasing sequences $\left\{f\left(y^{i}(t)\right)\right\}, i=1, \ldots, s$, and $\{f(\hat{y}(t))\}$ converge. Thus, it is reasonable to suppose that the expected values of $y^{i}(t), i=1, \ldots, s$, and $\hat{y}(t)$ converge too.

On the other hand, the difference between $\operatorname{proj}_{M_{t}}\left(x^{i}(t-1)+v^{i}(t)\right)$ and $x^{i}(t-1)+$ $v^{i}(t)-$ and thus between their expected values - is a multiple of $\alpha(t)$ for some choices of $D$. This situation occurs in coordinate search, where $D=D_{\oplus}$. Since there is a subsequence of the mesh size parameters that converges to zero, there is at least the guarantee that the expected difference between $x^{i}(t-1)+v^{i}(t)$ and its projection onto $M_{t}$ converges to zero in that subsequence.

Fig. 1 Plot of $\imath$ for $\mu \bar{\omega}_{1}=\nu \bar{\omega}_{2}=0.25$

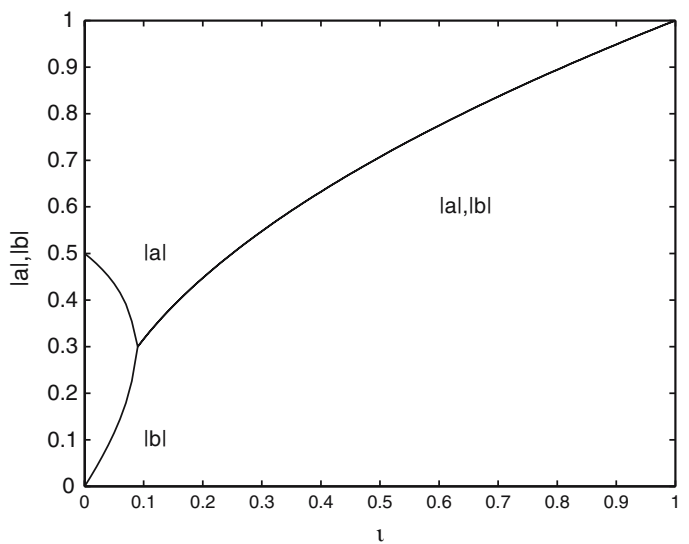


So, there is at least some indication that the term $g_{t}$ in (6) converges to zero for a subsequence of the iterates. Although this is not the same as saying that the assumptions of Theorem 5.2 are satisfied, it helps to explain the observed numerical termination of the algorithm.

\section{Optimization solvers used for comparison}

The particle swarm pattern search algorithm (Algorithm 4.1) was implemented in the $\mathrm{C}$ programming language. The solver is referred to as PSwarm.

In order to assess the performance of PSwarm, a set of 122 global optimization test problems from the literature $([2,17,21,25,28,29,32,34])$ was collected and coded in AMPL (see Table 2). All coded problems have lower and upper bounds on the variables. The problems description and their source are available at http : / / www . norg . uminho.pt/aivaz under software.

AMPL [14] is a mathematical modeling language which allows an easy and fast way to code optimization problems. AMPL provides also automatic differentiation (not used in the context of derivative free optimization) and interfaces to a number of optimization solvers. The AMPL mechanism to tune solvers was used to pass options to PSwarm.

\subsection{Solvers}

The optimization solver PSwarm was compared to a few solvers for global optimization, namely DIRECT [13], ASA [20], MCS [19], and PGAPack [27].

DIRECT is an implementation of the method described in ref. [23]. DIRECT reads from DIviding RECTangles and it is implemented in MATLAB. (In our numerical tests we used MATLAB Version 6, Release 12.) DIRECT solved the problems coded in AMPL by using the ampl func external AMPL function [15] for MATLAB together with a developed M-file to interface DIRECT with AMPL.

ASA is an implementation in C of the Adaptative Simulated Annealing. The user has to write its objective function as a $\mathrm{C}$ function and to compile it with the optimization code. Options are defined during compilation time. To use the AMPL coded problems, an interface for AMPL was also developed. Here, we have followed the ideas of the MATLAB interface to ASA provided by S. Sakata (see http: / / www. econ. 1sa. umich. edu/ sakata/software).

MCS stands for Multilevel Coordinate Search and it is inspired by the methods of Jones et al. [23]. MCS is implemented in MATLAB and, as with DIRECT, the AMPL interface to MATLAB and a developed M-file were used to obtain the numerical results for the AMPL coded problems.

PGAPack is an implementation of a genetic algorithm. The Parallel Genetic Algorithm Pack is written in C. As in ASA, the user defines a $\mathrm{C}$ function and compiles it along with the optimization code. As for ASA, an interface to AMPL was also developed. The population size selected for PGAPACk was changed to 200 (since it performed better with a higher population size).

DIRECT and MCS are deterministic codes. The other two, PGAPack and ASA, together with PSwarm, are stochastic ones. A relevant issue in stochastic codes is the choice of the underlying random number generator. It is well known that good random 
number generators are hard to find (see, for example, [33]). ASA and Pswarm use the number generator from [8,33]. PGAPack uses a generator described in ref. [22].

\subsection{PSwarm details}

The default values for PSwarm are $\alpha_{\mathrm{tol}}=10^{-5}, v=\mu=0.5, \alpha(0)=\max _{j=1, . ., n}\left(u_{j}-\right.$ $\left.\ell_{j}\right) / c$ with $c=5$, and $s=20$. The reduction of the mesh size parameter used $\theta(t)=0.5$. The expansion was only applied when two consecutive polls steps occured using the same polling direction [18]; in these cases we set $\phi(t)=2$. Polling was implemented in the opportunistic way (accepting the first polling point that yielded decrease). The projection onto the mesh $\left(\hat{x}^{i}(t)=\operatorname{proj}_{M_{t}}\left(x^{i}(t)\right)\right)$ has not been implemented in the search step.

The inertial parameter $\iota(t)$ is linearly interpolated between 0.9 and 0.4 , i.e., $\iota(t)=$ $0.9-\left(0.5 / t_{\max }\right) t$, where $t_{\max }$ is the maximum number of iterations permitted. Larger values for $\max \{|a|,|b|\}$ will result in slower convergence. Thus, we start with a slower rate and terminate faster.

The initial swarm is obtained by generating $s$ random feasible points uniformly distributed on $(\ell, u)$.

In particle swarm all particles will in principle converge to $\hat{y}$, and a high concentration of particles is needed in order to obtain a solution with some degree of precision. Thus, in the last iterations of particle swarm, a high number of objective function evaluations is necessary to obtain some precision in the solution. Removing particles from the swarm that are near others seems like a good idea, but a price in precision is paid in order to gain a decrease in the number of objective function evaluations.

In the proposed particle swarm pattern search algorithm the scenario is somehow different since the $\hat{y}$ particle position is improved by the poll steps of pattern search. Removing particles that do not drive the search to a global minimizer is highly desirable. A particle $i$ is removed from the swarm in PSwarm when it is close to $\hat{y}$, i.e., when $\left\|y^{i}(t)-\hat{y}(t)\right\| \leq \alpha(0)$. If a particle is close to $\hat{y}$ (compared in terms of $\alpha(0)$ ) it means that it is unlikely to further explore the search space for a global optimum.

\subsection{Performance profiles}

A fair comparison among different solvers should be based on the number of function evaluations, instead of based on the number of iterations or on the CPU time. The number of iterations is not a reliable measure because the amount of work done in each iteration is completely different among solvers, since some are population based and other are single point based. Since the quality of the solution is also an important measure of performance, the approach taken here consists of comparing the objective function values after a specified number of function evaluations.

The ASA solver does not support an option that limits the number of objective function evaluations. The interface developed for AMPL accounts for the number of objective function calls, and when the limit is reached exit is immediately forced by properly setting an ASA option. Solvers PSwarm, DIRECT, MCS, and PGaPack take control of the number of objective function evaluations in each iteration, and therefore the maximum number of objective function evaluations can be directly imposed. Algorithms that perform more than one objective function evaluation per iteration can exceed the requested maximum since the stopping criterion is checked at the beginning of each iteration. For instance, MCS for problem 1j1_38 (a Lennard-Jones 
cluster problem of size 38) computes 14,296 times the objective function value, when a maximum of 1,000 function evaluations is requested. Tuning other solvers options could reduce this gap, but we decide to use, as much as possible, the solver default options (the exceptions were the maximum numbers of function evaluations and iterations and the population size in PGAPack).

We present the numerical results in the form of performance profiles, as described in ref. [11]. This procedure was developed to benchmark optimization software, i.e., to compare different solvers on several (possibly many) test problems. One advantage of the performance profiles is that they can be presented in one figure, by plotting for the different solvers a cumulative distribution function $\rho(\tau)$ representing a performance ratio.

The performance ratio is defined by first setting $r_{p, s}=\frac{t_{p, s}}{\min \left\{t_{p, s}: s \in \mathcal{S}\right\}}, p \in \mathcal{P}, s \in \mathcal{S}$, where $\mathcal{P}$ is the test set, $\mathcal{S}$ is the set of solvers, and $t_{p, s}$ is the value obtained by solver $s$ on test problem $p$. Then, define $\rho_{s}(\tau)=\frac{1}{n_{p}} \operatorname{size}\left\{p \in \mathcal{P}: r_{p, s} \leq \tau\right\}$, where $n_{p}$ is the number of test problems. The value of $\rho_{s}(1)$ is the probability that the solver will win over the remaining ones (meaning that it will yield a value lower than the values of the remaining ones). If we are only interested in determining which solver is the best (in the sense that wins the most), we compare the values of $\rho_{S}(1)$ for all the solvers. At the other end, solvers with the largest probabilities $\rho_{s}(\tau)$ for large values of $\tau$ are the most robust ones (meaning that are the ones that solved more problems).

The performance profile measure described in ref. [11] was the computing time required to solve the problem, but other performance quantities can be used, such as the number of function evaluations. However, the objective function value achieved at the maximum number of function evaluations imposed cannot be used directly as a performance profile measure. For instance, a problem in the test set whose objective function value at the solution computed by one of the solvers is zero could lead to $\min \left\{t_{p, s}: s \in \mathcal{S}\right\}=0$. If the objective function value at the solution determined by a solver is negative, then the value of $r_{p, s}$ could also be negative. In any of these situations, it is not possible to use the performance profiles.

For each stochastic solver, several runs must be made for every problem, so that average, best, and worst behavior can be analyzed. In ref. [2], the following scaled performance profile measure

$$
t_{p, s}=\frac{\bar{f}_{p, s}-f_{p}^{*}}{f_{p, w}-f_{p}^{*}}
$$

was introduced, where $\bar{f}_{p, s}$ is the average objective function value obtained for the runs of solver $s$ on problem $p, f_{p}^{*}$ is the best function value found among all the solvers (or the global minimum when known), and $f_{p, w}$ is the worst function value found among all the solvers. If we were interested in the best (worst) behavior we would use, instead of $\bar{f}_{p, s}$, the best (worst) value among all runs of the stochastic solver $s$ on problem $p$.

While using (10) could prevent $r_{p, s}$ from taking negative values, a division by zero can occur when $f_{p, w}=f_{p}^{*}$. To avoid this, we suggest a shift to the positive axis for problems where a negative or zero $\min \left\{t_{p, s}: s \in \mathcal{S}\right\}$ is obtained. Our performance profile measure is defined as:

$t_{p, s}=$ (best/average/worst) objective function value obtained for problem $p$ by solver $s$ (for all runs if solver $s$ is stochastic), 


$$
r_{p, s}= \begin{cases}1+t_{p, s}-\min \left\{t_{p, s}: s \in \mathcal{S}\right\}, & \text { when } \min \left\{t_{p, s}: s \in \mathcal{S}\right\}<\epsilon, \\ \frac{t_{p, s}}{\min \left\{t_{p, s}: s \in \mathcal{S}\right\}}, & \text { otherwise. }\end{cases}
$$

We set $\epsilon=0.001$.

\section{Numerical results}

All tests were run in a Pentium IV $(3.0 \mathrm{GHz}$ and $1 \mathrm{~Gb}$ of RAM). Stochastic solvers PSwarm, ASA, and PGAPack were run 30 times, while the deterministic solvers DIRECT and MCS were run only once.

Figures 2-4 are performance profile plots for the best, average, and worst solutions found, respectively, when the maximum number of function evaluations $(\max f)$ was set to 1,000 for problems with dimension lower than 100 , and to 7,500 for the 13 remaining ones. We will denote this by $\max f=1,000(7,500)$. Figures $5-7$ correspond to Figs. $2-4$, respectively, but when $\max f=10,000(15,000)$. Each figure includes two plots: one for better visibility around $\rho(1)$ and the other to capture the tendency near $\rho(\infty)$.

From Fig. 2, we can conclude that PSwarm has a slight advantage over the other solvers in the best behavior case for $\max f=1,000(7,500)$. In the average and worst behaviors, PSwarm loses in performance against DIRECT and MCS. In any case, it wins against the other solvers with respect to robustness.

When $\max f=10,000(15,000)$ and for the best behavior, PSwarm and MCS perform better than the other solvers, the former being slightly more robust. In the average and worst scenarios, PSwarm loses against DIRECT and MCS, but wins on robustness overall.

In Figs. 8 and 9, we plot the profiles for the number of function evaluations taken to solve the problems in our list for the cases where $\max f=1,000$ and $\max f=10,000$.
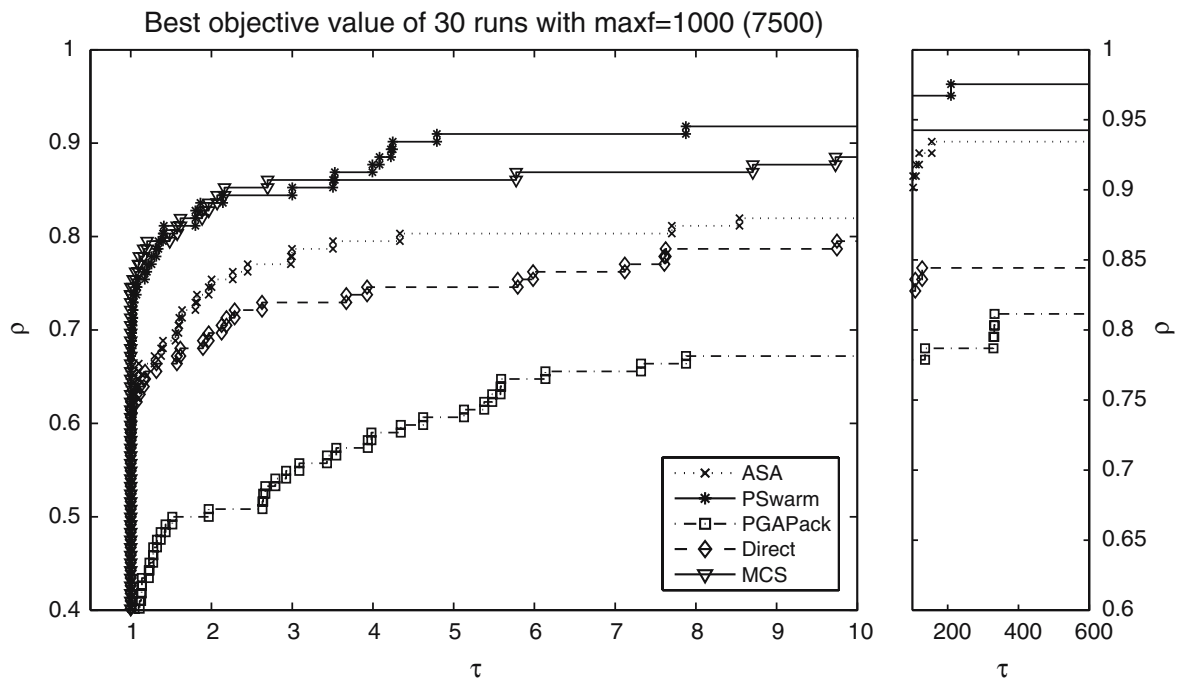

Fig. 2 Best objective function value for 30 runs with $\max f=1,000(7,500)$ 

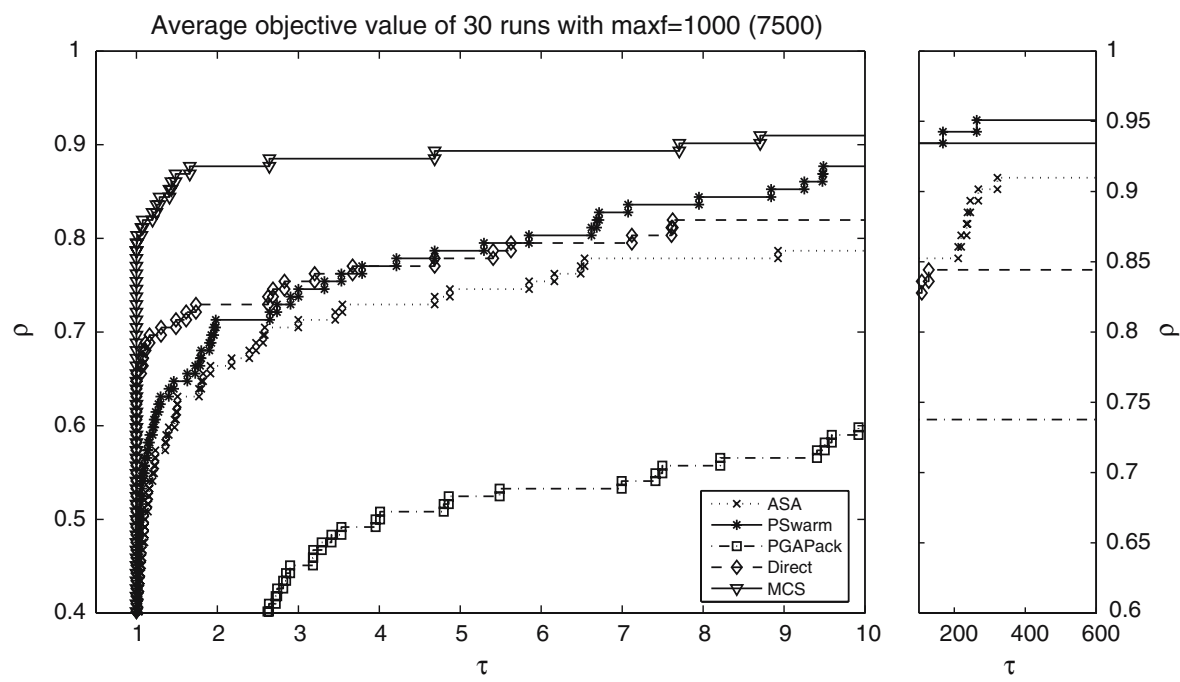

Fig. 3 Average objective function value for 30 runs with $\max f=1,000(7,500)$
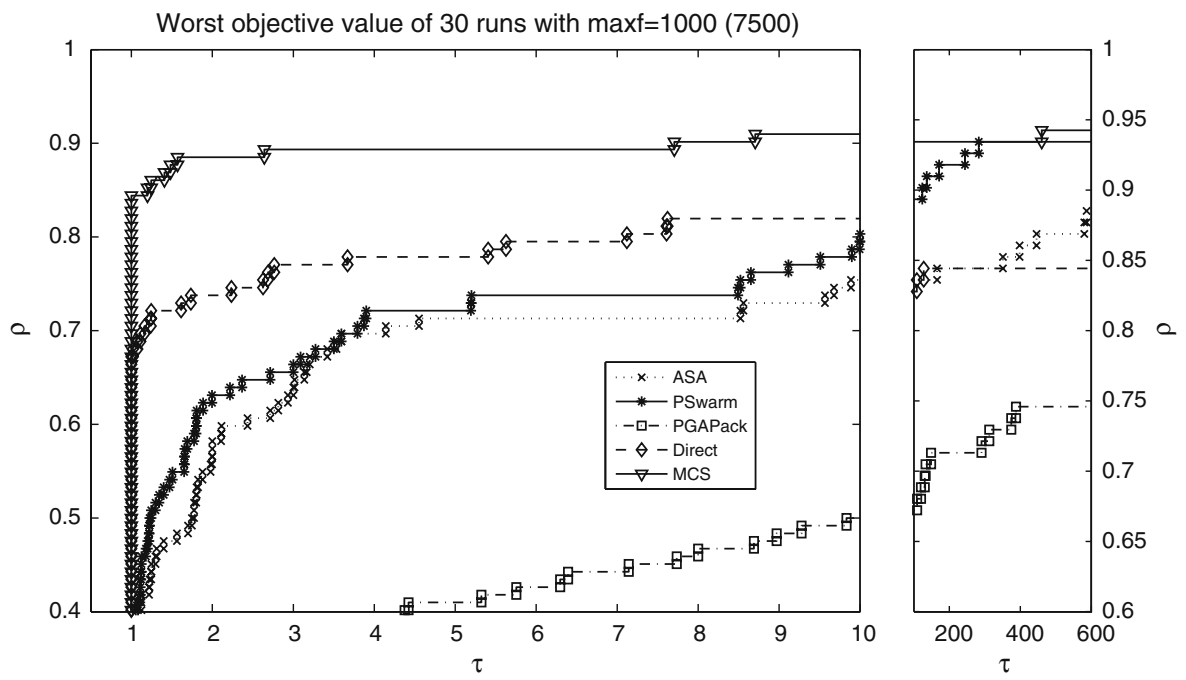

Fig. 4 Worst objective function value for 30 runs with $\max f=1,000(7,500)$

The best solver is MCS, which is not surprising since it is based on interpolation models and most of the objective functions tested are smooth. PSwarm appears clearly in second place in these profiles. Moreover, in Table 1 we report the corresponding average number of function evaluations. One can see from these tables that PSwarm appears first and MCS performed apparently worse. This effect is due to some of the problems in our test set where the objective function exhibits steep oscillations. PSwarm is a direct search type method and thus better suited to deal with these types of functions, and thus it seemed to present the best balance (among all solvers) for smooth and less smooth types of objective functions. 

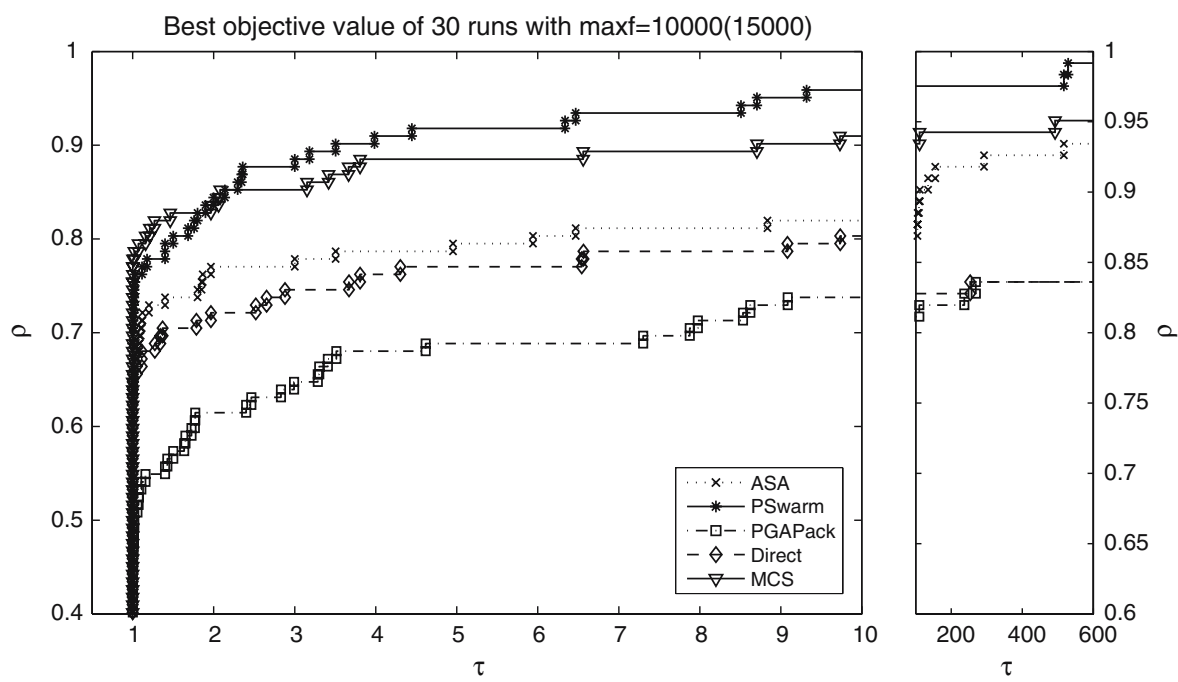

Fig. 5 Best objective function value for 30 runs with $\max f=10,000(15,000)$
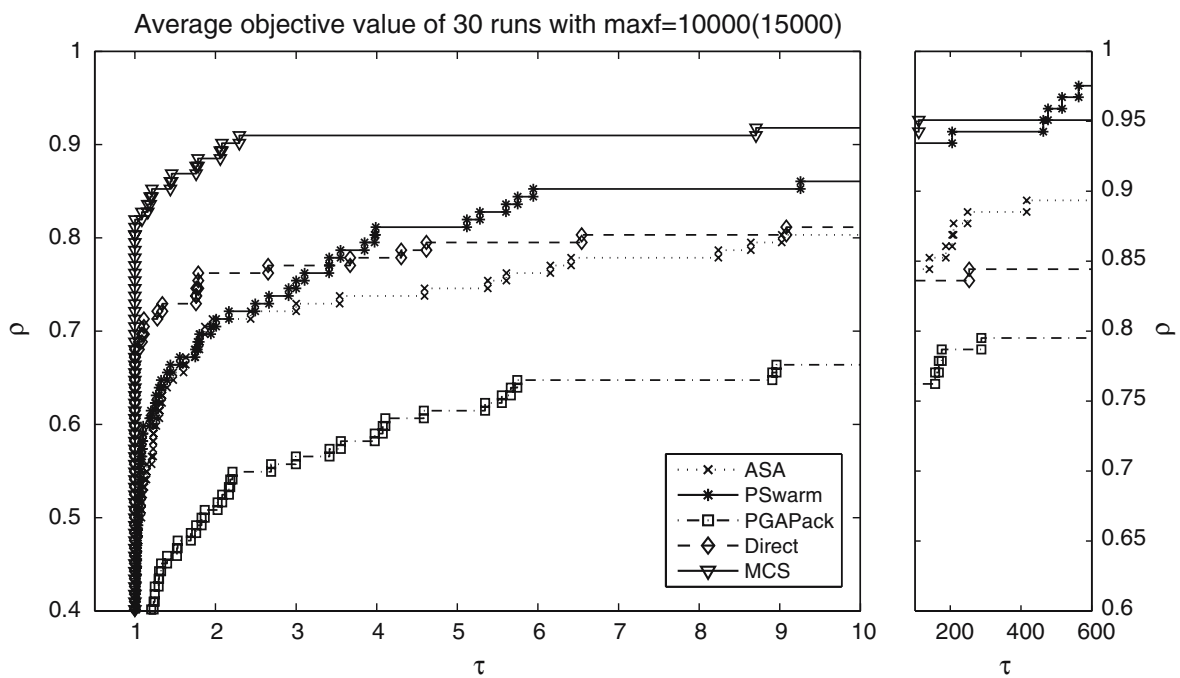

Fig. 6 Average objective function value for 30 runs with $\max f=10,000(15,000)$

It is important to point out that the performance of DIRECT is not necessarily better than the one of PSwarm, a conclusion which could be wrongly drawn from the profiles for the quality of the final objective value. In fact, the stopping criterion for DIRECT (as well as for PGAPack) is based on the maximum number of function evaluations permitted. One can clearly see from Table 1 that PSwarm required fewer function evaluations than DIRECT or ASA.

Table 2 reports detailed numerical results obtained by the solver PSwarm for all problems in our test set. The maximum number of function evaluations was set to 10,000 . For each problem, we chose to report the best result (in terms of $f$ ) obtained 

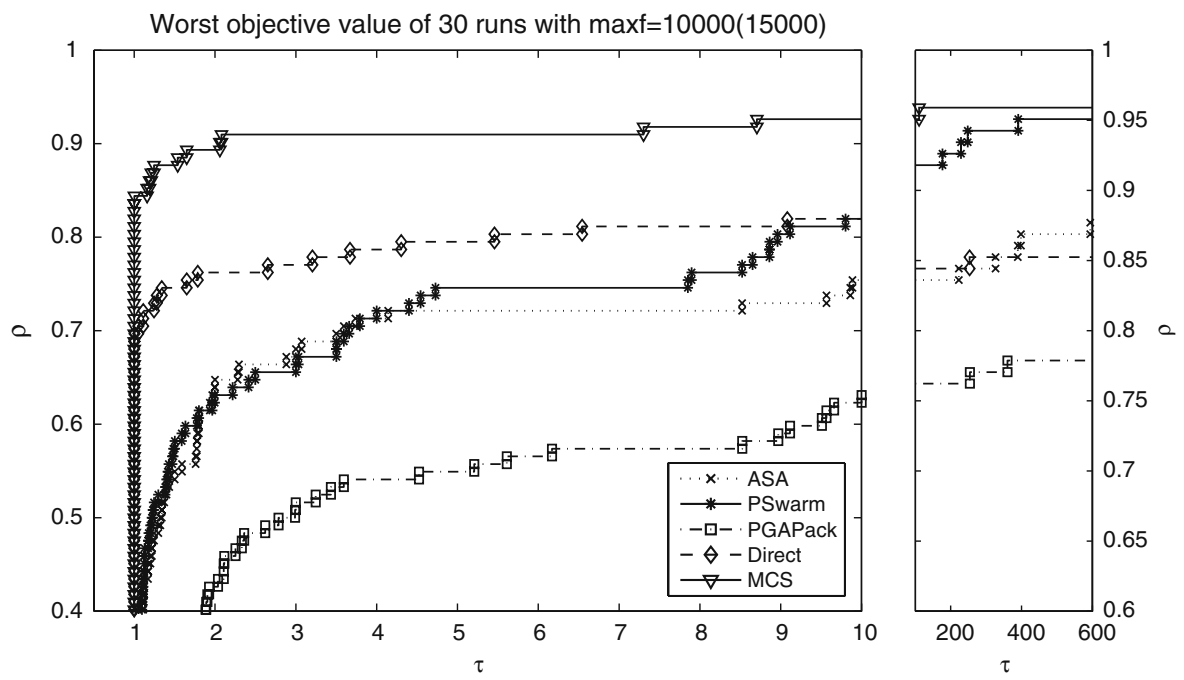

Fig. 7 Worst objective function value for 30 runs with $\max f=10,000(15,000)$
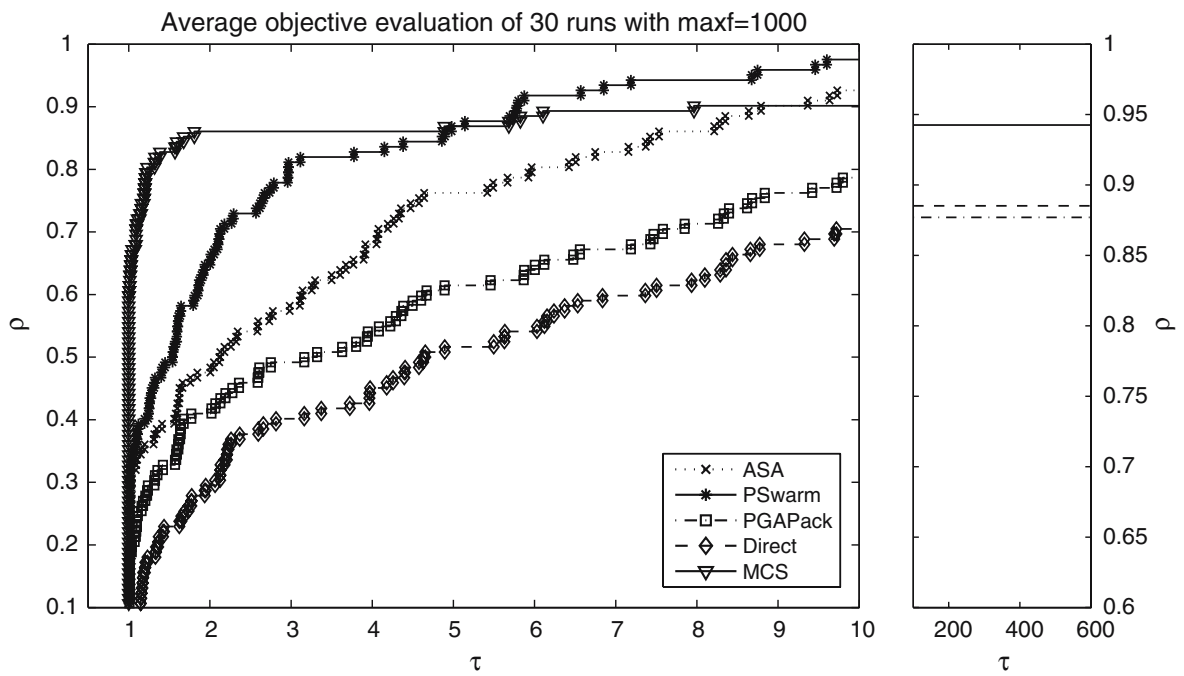

Fig. 8 Number of objective function evaluations in the case $\max f=1,000$ (averages among the 30 runs for stochastic solvers)

among the 30 runs. The columns in the table refer to: problem name; AMPL model file (problem); number of variables $(n)$; number of objective function evaluations (nfevals); number of iterations (niter); number of poll steps (npoll); percentage of successful poll steps (\%spoll); optimality gap when known; otherwise the value marked with $*$ is just the final objective function calculated (gap). We did not report the final number of particles because this number is equal to one in the majority of the problems ran. 

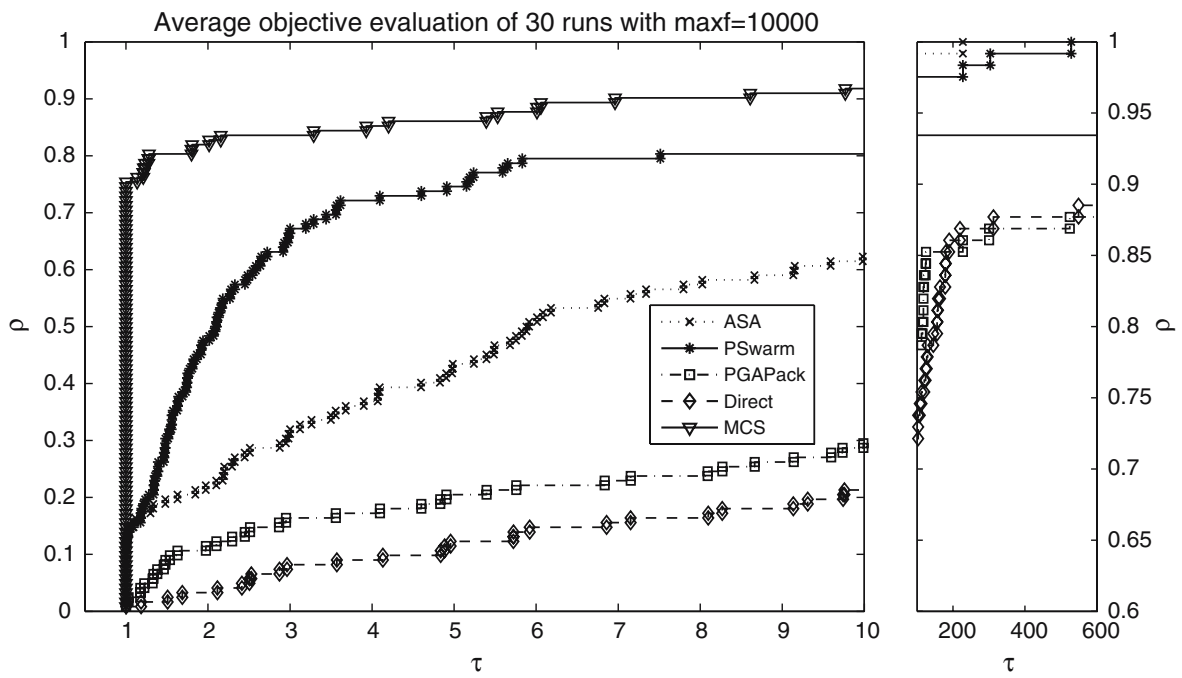

Fig. 9 Number of objective function evaluations in the case $\max f=10,000$ (averages among the 30 runs for stochastic solvers)

Table 1 Average number of function evaluations for the test set in the cases $\max f=1,000$ and $\max f=10,000$ (averages among the 30 runs for stochastic solvers)

\begin{tabular}{lccccc}
\hline $\max f$ & ASA & PGAPack & PSwarm & DIRECT & MCS \\
\hline 1,000 & 857 & 1,009 & 686 & 1,107 & 1,837 \\
10,000 & 5,047 & 10,009 & 3,603 & 11,517 & 4,469 \\
\hline
\end{tabular}

Using the same test set, we have also compared PSwarm against implementations of the coordinate search algorithm (denoted by PS) and the particle swarm algorithm (denoted by PSOA). These last two algorithms were reported in Sects. 2 and 3, respectively. We recall that PSwarm is a combination of PS and PSOA. We used the same parameters in PS and PSOA as in PSwarm. The corresponding profiles are shown in Figs. 10 and 11 for the average values of 30 runs (in the PSOA and PSwarm cases). As expected, coordinate search performed worse since it might got stuck around local minimizers or stationary points with lower objective function values. The performance of particle swarm alone is good although worse than the one of PSwarm, which seems to have gained in robustness by incorporating coordinate search.

\section{Conclusions and future work}

In this paper, we developed a hybrid algorithm for global minimization subject to simple bounds that combines a heuristic for global optimization (particle swarm) with a rigorous method (pattern search) for local minimization. The proposed particle swarm pattern search method enjoys the global convergence properties (i.e., from any starting point) of pattern search to stationary points. 
Table 2 Numerical results obtained by PSwarm

\begin{tabular}{|c|c|c|c|c|c|c|}
\hline problem & $n$ & nfevals & niter & npoll & \%spoll & gap \\
\hline ack & 10 & 1,797 & 121 & 117 & 81.2 & $2.171640 \mathrm{E}-01$ \\
\hline ap & 2 & 207 & 34 & 32 & 40.63 & $-8.600000 \mathrm{E}-05$ \\
\hline bf1 & 2 & 204 & 36 & 33 & 33.33 & $0.000000 \mathrm{E}+00$ \\
\hline bf2 & 2 & 208 & 37 & 35 & 37.14 & $0.000000 \mathrm{E}+00$ \\
\hline bhs & 2 & 218 & 29 & 28 & 39.29 & $-1.384940 \mathrm{E}-01$ \\
\hline bl & 2 & 217 & 36 & 34 & 41.18 & $0.000000 \mathrm{E}+00$ \\
\hline bp & 2 & 224 & 39 & 37 & 45.95 & $-3.577297 \mathrm{E}-07$ \\
\hline cb3 & 2 & 190 & 29 & 27 & 29.63 & $0.000000 \mathrm{E}+00$ \\
\hline cb6 & 2 & 211 & 37 & 35 & 48.57 & $-2.800000 \mathrm{E}-05$ \\
\hline $\mathrm{cm} 2$ & 2 & 182 & 34 & 31 & 45.16 & $0.000000 \mathrm{E}+00$ \\
\hline $\mathrm{cm} 4$ & 4 & 385 & 45 & 41 & 60.98 & $0.000000 \mathrm{E}+00$ \\
\hline da & 2 & 232 & 45 & 41 & 48.78 & $4.816600 \mathrm{E}-01$ \\
\hline em_10 & 10 & 4,488 & 324 & 321 & 89.41 & $1.384700 \mathrm{E}+00$ \\
\hline em_5 & 5 & 823 & 99 & 94 & 79.79 & $1.917650 \mathrm{E}-01$ \\
\hline ep.mod & 2 & 227 & 39 & 35 & 45.71 & $0.000000 \mathrm{E}+00$ \\
\hline exp.mod & 10 & 1,434 & 84 & 80 & 80 & $0.000000 \mathrm{E}+00$ \\
\hline fls.mod & 2 & 227 & 28 & 22 & 27.27 & $3.000000 \mathrm{E}-06$ \\
\hline fr.mod & 2 & 337 & 71 & 67 & 52.24 & $0.000000 \mathrm{E}+00$ \\
\hline fx_10 & 10 & 1,773 & 125 & 108 & 78.7 & $8.077291 \mathrm{E}+00$ \\
\hline fx_5 & 5 & 799 & 123 & 57 & 68.42 & $6.875980 \mathrm{E}+00$ \\
\hline $\mathrm{gp}$ & 2 & 190 & 28 & 26 & 30.77 & $0.000000 \mathrm{E}+00$ \\
\hline grp & 3 & 1,339 & 263 & 28 & 28.57 & $0.000000 \mathrm{E}+00$ \\
\hline gw & 10 & 2,296 & 152 & 146 & 82.19 & $0.000000 \mathrm{E}+00$ \\
\hline h3 & 3 & 295 & 37 & 35 & 57.14 & $0.000000 \mathrm{E}+00$ \\
\hline h6 & 6 & 655 & 59 & 51 & 68.63 & $0.000000 \mathrm{E}+00$ \\
\hline $\mathrm{hm}$ & 2 & 195 & 32 & 30 & 36.67 & $0.000000 \mathrm{E}+00$ \\
\hline $\mathrm{hm} 1$ & 1 & 96 & 22 & 20 & 15 & $0.000000 \mathrm{E}+00$ \\
\hline $\mathrm{hm} 2$ & 1 & 141 & 29 & 27 & 25.93 & $-1.447000 \mathrm{E}-02$ \\
\hline $\mathrm{hm} 3$ & 1 & 110 & 22 & 21 & 19.05 & $2.456000 \mathrm{E}-03$ \\
\hline $\mathrm{hm} 4$ & 2 & 198 & 31 & 28 & 35.71 & $0.000000 \mathrm{E}+00$ \\
\hline $\mathrm{hm} 5$ & 3 & 255 & 34 & 30 & 50 & $0.000000 \mathrm{E}+00$ \\
\hline hsk & 2 & 204 & 28 & 26 & 34.62 & $-1.200000 \mathrm{E}-05$ \\
\hline hv & 3 & 343 & 44 & 42 & 54.76 & $0.000000 \mathrm{E}+00$ \\
\hline ir0 & 4 & 671 & 84 & 80 & 66.25 & $0.000000 \mathrm{E}+00$ \\
\hline ir1 & 3 & 292 & 41 & 37 & 51.35 & $0.000000 \mathrm{E}+00$ \\
\hline ir2 & 2 & 522 & 131 & 119 & 61.34 & $1.000000 \mathrm{E}-06$ \\
\hline ir3 & 5 & 342 & 25 & 20 & 10 & $0.000000 \mathrm{E}+00$ \\
\hline ir4 & 30 & 8,769 & 250 & 244 & 93.03 & $1.587200 \mathrm{E}-02$ \\
\hline ir5 & 2 & 513 & 116 & 40 & 45 & $1.996000 \mathrm{E}-03$ \\
\hline $\mathrm{kl}$ & 4 & 1,435 & 170 & 164 & 75.61 & $-4.800000 \mathrm{E}-07$ \\
\hline ks & 1 & 92 & 18 & 17 & 0 & $0.000000 \mathrm{E}+00$ \\
\hline lj1_38 & 114 & 10,072 & 146 & 127 & 95.28 & $1.409238 \mathrm{E}+02^{*}$ \\
\hline lj1_75 & 225 & 10,063 & 137 & 127 & 96.85 & $3.512964 \mathrm{E}+04^{*}$ \\
\hline lj1_98 & 294 & 10,072 & 129 & 119 & 98.32 & $1.939568 \mathrm{E}+05^{*}$ \\
\hline lj2_38 & 114 & 10,109 & 153 & 139 & 95.68 & $3.727664 \mathrm{E}+02^{*}$ \\
\hline lj2_75 & 225 & 10,090 & 116 & 90 & 98.89 & $3.245009 \mathrm{E}+04^{*}$ \\
\hline lj2_98 & 294 & 10,036 & 125 & 114 & 98.25 & $1.700452 \mathrm{E}+05^{*}$ \\
\hline lj3_38 & 114 & 10,033 & 157 & 127 & 93.7 & $1.729289 \mathrm{E}+03^{*}$ \\
\hline lj3_75 & 225 & 10,257 & 124 & 112 & 98.21 & $1.036894 \mathrm{E}+06^{*}$ \\
\hline lj3_98 & 294 & 10,050 & 113 & 107 & 99.07 & $1.518801 \mathrm{E}+07^{*}$ \\
\hline $\operatorname{lm} 1$ & 3 & 335 & 44 & 40 & 52.5 & $0.000000 \mathrm{E}+00$ \\
\hline $\operatorname{lm} 2 \_10$ & 10 & 1,562 & 93 & 86 & 77.91 & $0.000000 \mathrm{E}+00$ \\
\hline $\operatorname{lm} 2 \_5$ & 5 & 625 & 59 & 56 & 67.86 & $0.000000 \mathrm{E}+00$ \\
\hline $\operatorname{lms} 1 \mathrm{a}$ & 2 & 1,600 & 172 & 123 & 55.28 & $-2.000000 \mathrm{E}-06$ \\
\hline $\operatorname{lms} 1 \mathrm{~b}$ & 2 & 2,387 & 452 & 55 & 36.36 & $1.078700 \mathrm{E}-02$ \\
\hline $\operatorname{lms} 2$ & 3 & 1,147 & 163 & 60 & 48.33 & $1.501300 \mathrm{E}-02$ \\
\hline
\end{tabular}


Table 2 continued

\begin{tabular}{|c|c|c|c|c|c|c|}
\hline problem & $n$ & nfevals & niter & npoll & $\%$ spoll & gap \\
\hline $\operatorname{lms} 3$ & 4 & 2,455 & 262 & 109 & 53.21 & $6.233700 \mathrm{E}-02$ \\
\hline $\operatorname{lms} 5$ & 6 & 5,596 & 1,631 & 366 & 59.84 & 7.384100E-02 \\
\hline lv8 & 3 & 310 & 42 & 39 & 48.72 & $0.000000 \mathrm{E}+00$ \\
\hline $\mathrm{mc}$ & 2 & 211 & 32 & 29 & 41.38 & 7.700000E-05 \\
\hline $\mathrm{mcp}$ & 4 & 248 & 29 & 22 & 27.27 & $0.000000 \mathrm{E}+00$ \\
\hline mgp & 2 & 193 & 33 & 31 & 41.94 & $-2.593904 \mathrm{E}+00$ \\
\hline mgw_10 & 10 & 10,007 & 473 & 461 & 93.71 & 1.107800E-02 \\
\hline mgw_2 & 2 & 339 & 43 & 37 & 43.24 & $0.000000 \mathrm{E}+00$ \\
\hline mgw_20 & 20 & 10,005 & 306 & 299 & 93.98 & $5.390400 \mathrm{E}-02$ \\
\hline ml_10 & 10 & 2,113 & 129 & 118 & 75.42 & $0.000000 \mathrm{E}+00$ \\
\hline ml_5 & 5 & 603 & 59 & 55 & 67.27 & $0.000000 \mathrm{E}+00$ \\
\hline $\mathrm{mr}$ & 3 & 886 & 179 & 171 & 62.57 & $1.860000 \mathrm{E}-03$ \\
\hline $\operatorname{mrp}$ & 2 & 217 & 44 & 43 & 55.81 & $0.000000 \mathrm{E}+00$ \\
\hline ms1 & 20 & 3,512 & 216 & 207 & 90.82 & $4.326540 \mathrm{E}-01$ \\
\hline $\mathrm{ms} 2$ & 20 & 3,927 & 238 & 225 & 91.56 & $-1.361000 \mathrm{E}-02$ \\
\hline $\mathrm{nf} 2$ & 4 & 2,162 & 205 & 198 & 64.65 & $2.700000 \mathrm{E}-05$ \\
\hline nf3_10 & 10 & 4,466 & 586 & 579 & 95.16 & $0.000000 \mathrm{E}+00$ \\
\hline nf3_15 & 15 & 10,008 & 800 & 792 & 96.46 & $7.000000 \mathrm{E}-06$ \\
\hline nf3_20 & 20 & 10,008 & 793 & 768 & 94.92 & $2.131690 \mathrm{E}-01$ \\
\hline nf3_25 & 25 & 10,025 & 535 & 508 & 95.67 & 5.490210E-01 \\
\hline nf3_30 & 30 & 10,005 & 359 & 347 & 96.25 & $6.108021 \mathrm{E}+01$ \\
\hline osp_10 & 10 & 1,885 & 134 & 121 & 80.17 & $1.143724 \mathrm{E}+00$ \\
\hline osp_20 & 20 & 5,621 & 229 & 220 & 90.45 & $1.143833 \mathrm{E}+00$ \\
\hline plj_38 & 114 & 10,103 & 163 & 135 & 96.3 & $7.746385 \mathrm{E}+02 *$ \\
\hline plj_75 & 225 & 10,028 & 127 & 109 & 98.17 & $3.728411 \mathrm{E}+04^{*}$ \\
\hline plj_98 & 294 & 10,182 & 119 & 105 & 98.1 & $1.796150 \mathrm{E}+05^{*}$ \\
\hline $\mathrm{pp}$ & 10 & 1,578 & 104 & 100 & 81 & $-4.700000 \mathrm{E}-04$ \\
\hline prd & 2 & 400 & 66 & 34 & 44.12 & $0.000000 \mathrm{E}+00$ \\
\hline ptm & 9 & 10,009 & 1186 & 618 & 73.46 & $3.908401 \mathrm{E}+00$ \\
\hline pwq & 4 & 439 & 57 & 53 & 60.38 & $0.000000 \mathrm{E}+00$ \\
\hline $\mathrm{rb}$ & 10 & 10,003 & 793 & 712 & 76.12 & $1.114400 \mathrm{E}-02$ \\
\hline rg_10 & 10 & 4,364 & 672 & 158 & 71.52 & $0.000000 \mathrm{E}+00$ \\
\hline rg_2 & 2 & 210 & 34 & 32 & 43.75 & $0.000000 \mathrm{E}+00$ \\
\hline s10 & 4 & 431 & 51 & 48 & 62.5 & $-4.510000 \mathrm{E}-03$ \\
\hline s5 & 4 & 395 & 46 & 43 & 58.14 & $-3.300000 \mathrm{E}-03$ \\
\hline s7 & 4 & 415 & 52 & 49 & 63.27 & $-3.041000 \mathrm{E}-03$ \\
\hline sal_10 & 10 & 1,356 & 76 & 68 & 60.29 & 3.998730E-01 \\
\hline sal_5 & 5 & 452 & 39 & 37 & 40.54 & 1.998730E-01 \\
\hline $\mathrm{sbt}$ & 2 & 305 & 39 & 37 & 45.95 & $-9.000000 \mathrm{E}-06$ \\
\hline sf1 & 2 & 210 & 32 & 29 & 24.14 & $9.716000 \mathrm{E}-03$ \\
\hline $\mathrm{sf} 2$ & 2 & 266 & 45 & 41 & 43.9 & $5.383000 \mathrm{E}-03$ \\
\hline shv1 & 1 & 101 & 20 & 19 & 21.05 & $-1.000000 \mathrm{E}-03$ \\
\hline $\operatorname{shv} 2$ & 2 & 196 & 33 & 31 & 41.94 & $0.000000 \mathrm{E}+00$ \\
\hline sin_10 & 10 & 1,872 & 124 & 117 & 81.2 & $0.000000 \mathrm{E}+00$ \\
\hline sin_20 & 20 & 5,462 & 225 & 216 & 88.43 & $0.000000 \mathrm{E}+00$ \\
\hline st_- 17 & 17 & 10,011 & 1,048 & 457 & 78.12 & $3.081935 \mathrm{E}+06$ \\
\hline st_9 & 9 & 10,001 & 1,052 & 847 & 82.88 & $7.516622 \mathrm{E}+00$ \\
\hline stg & 1 & 113 & 26 & 23 & 17.39 & $0.000000 \mathrm{E}+00$ \\
\hline swf & 10 & 2,311 & 161 & 158 & 82.91 & $1.184385 \mathrm{E}+02$ \\
\hline SZ & 1 & 125 & 34 & 28 & 25 & $-2.561249 E+00$ \\
\hline Szzs & 1 & 112 & 29 & 27 & 33.33 & $-1.308000 \mathrm{E}-03$ \\
\hline wf & 4 & 10,008 & 3,505 & 1,150 & 59.57 & $2.500000 \mathrm{E}-05$ \\
\hline xor & 9 & 887 & 73 & 60 & 68.33 & 8.678270E-01 \\
\hline zkv_10 & 10 & 10,003 & 1,405 & 752 & 75.8 & $1.393000 \mathrm{E}-03$ \\
\hline $\mathrm{zkv \_ 2}$ & 2 & 212 & 39 & 35 & 45.71 & $0.000000 \mathrm{E}+00$ \\
\hline zkv_20 & 20 & 10,018 & 1,031 & 422 & 77.01 & $3.632018 \mathrm{E}+01$ \\
\hline
\end{tabular}


Table 2 continued

\begin{tabular}{llrrrlr}
\hline problem & $n$ & nfevals & niter & npoll & \% spoll & \multicolumn{1}{l}{ gap } \\
\hline zkv_5 & 5 & 1,318 & 168 & 163 & 85.89 & $0.000000 \mathrm{E}+00$ \\
zlk1 & 1 & 119 & 27 & 25 & 20 & $4.039000 \mathrm{E}-03$ \\
zlk2a & 1 & 130 & 26 & 22 & 22.73 & $-5.000000 \mathrm{E}-03$ \\
zlk2b & 1 & 113 & 26 & 24 & 25 & $-5.000000 \mathrm{E}-03$ \\
zlk3a & 1 & 138 & 32 & 29 & 24.14 & $0.000000 \mathrm{E}+00$ \\
zlk3b & 1 & 132 & 32 & 29 & 24.14 & $0.000000 \mathrm{E}+00$ \\
zlk3c & 1 & 132 & 27 & 25 & 24 & $0.000000 \mathrm{E}+00$ \\
zlk4 & 2 & 224 & 39 & 37 & 45.95 & $-2.112000 \mathrm{E}-03$ \\
zlk5 & 3 & 294 & 40 & 37 & 56.76 & $-2.782000 \mathrm{E}-03$ \\
zzs & 1 & 120 & 29 & 26 & 23.08 & $-4.239000 \mathrm{E}-03$ \\
\hline
\end{tabular}
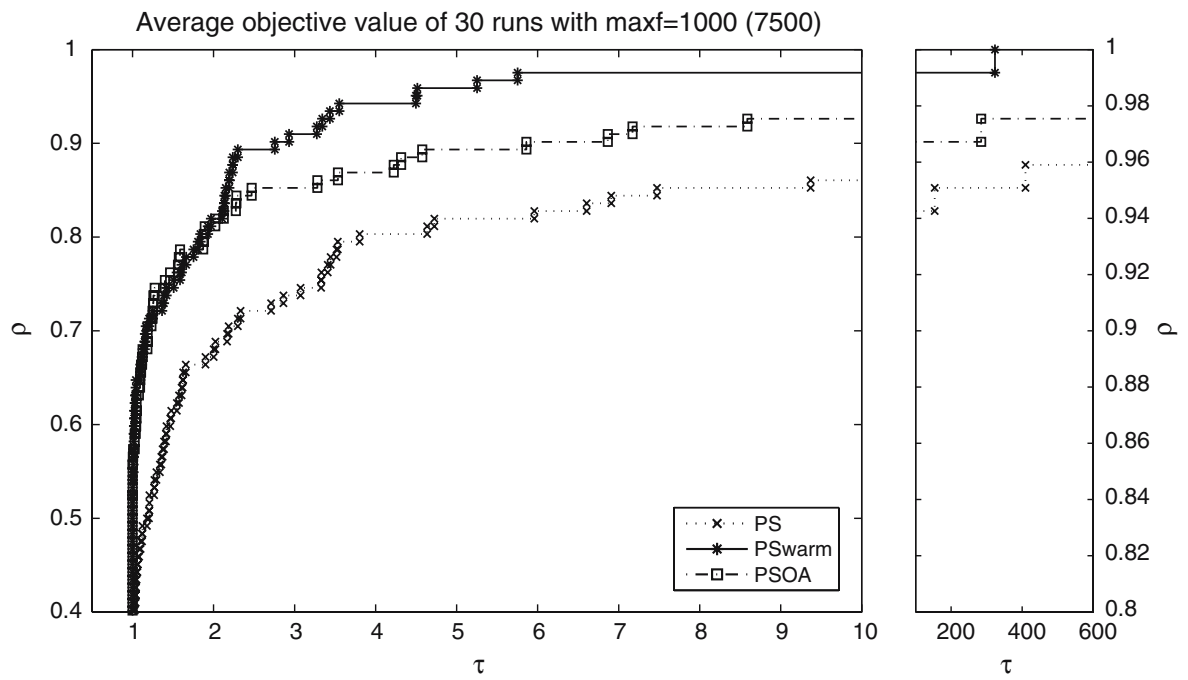

Fig. 10 Average objective function value for 30 runs with $\max f=1,000(1,500)$

We presented some analysis for the particle swarm pattern search method that indicates proper termination for an appropriate hybrid stopping criterion. The numerical results are particularly encouraging given that no fine tuning of algorithmic choices or parameters has been done yet for the new algorithm. A basic implementation of the particle swarm pattern search (PSwarm solver) has been shown to be the most robust among all global optimization solvers tested and to be highly competitive in efficiency with the most efficient of these solvers (MCS).

We plan to implement the particle swarm pattern search method in a parallel environment, since both techniques (particle swarm and pattern search) are easy to parallelize. In the search step of the method, where particle swarm is applied, one can distribute the evaluation of the objective function on the new swarm by the processors available. The same can be done in the poll step for the poll set. Another task for future research is to handle problems with more general type of constraints. Other research avenues can be considered when a cheaper surrogate for the function $f$ is available. For instance, one can consider the application of particle swarm in the search step to the surrogate itself rather than to the true function. 

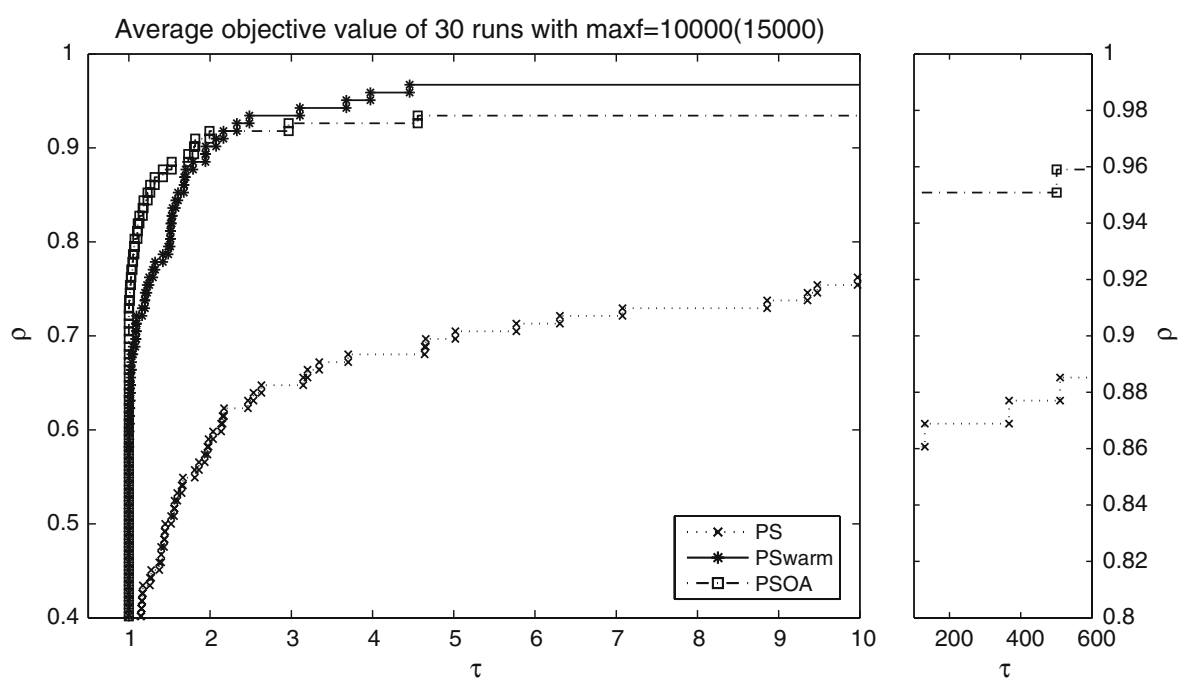

Fig. 11 Average objective function value for 30 runs with $\max f=10,000(15,000)$

Acknowledgements The authors are grateful to Montaz Ali, Joerg M. Gablonsky, Arnold Neumaier, and two anonymous referees for their comments and suggestions.

\section{References}

1. Alberto, P., Nogueira, F., Rocha, H., Vicente, L.N.: Pattern search methods for user-provided points: application to molecular geometry problems. SIAM J. Optim. 14, 1216-1236 (2004)

2. Ali, M.M., Khompatraporn, C., Zabinsky, Z.B.: A numerical evaluation of several stochastic algorithms on selected continuous global optimization test problems. J. Global Optim. 31, 635-672 (2005)

3. Audet, C., Dennis, J.E.: Analysis of generalized pattern searches. SIAM J. Optim. 13, 889-903 (2003)

4. Audet, C., Dennis, J.E.: Mesh adaptive direct search algorithms for constrained optimization. SIAM J. Optim. 17, 188-217 (2006)

5. Audet, C., Orban, D.: Finding optimal algorithmic parameters using derivative-free optimization. SIAM J. Optim. 17, 642-664 (2006)

6. van den Bergh, F.: An analysis of particle swarm optimizers. Ph.D thesis, Faculty of Natural and Agricultural Science, University of Pretoria (2001)

7. Van Den Berghand, F., Engelbrecht, A.P.: A study of particle swarm optimization particle trajectories. Inf. Sci. 176, 937-971 (2006)

8. Binder, A.K., Stauffer, A.D.: A simple introduction to Monte Carlo simulations and some specialized topics. In: Binder, E.K. (ed.) Applications of the Monte Carlo Method in Statistical Physics, pp. 1-36. Springer, Berlin Heidelberg New York (1985)

9. Custódio, A.L., Vicente, L.N.: Using sampling and simplex derivatives in pattern search methods. SIAM J. Optim. (2007, in press)

10. Davis, C.: Theory of positive linear dependence. Am. J. Math. 76, 733-746 (1954)

11. Dolan, E.D., Moré, J.J.: Benchmarking optimization software with performance profiles. Math. Program. 91, 201-213 (2002)

12. Eberhart, R., Kennedy, J.: New optimizers using particle swarm theory. In: Proceedings of the 1995 6th International Symposium on Micro Machine and Human Science, pp. 39-43, Nagoya, Japan. IEEE Service Center, Piscata way, NJ (1995)

13. Finkel, D.E.: DIRECT Optimization Algorithm User Guide. North Carolina State University (2003) http://www4.ncsu.edu/ definkel/research/index.html 
14. Fourer, R., Gay, D.M., Kernighan, B.W.: A modeling language for mathematical programming. Manage. Sci. 36, 519-554 (1990)

15. Gay, D.M.: Hooking your solver to AMPL. Numerical Analysis Manuscript 93-10, AT\&T Bell Laboratories (1993) http://www.ampl.com

16. Hart, W.E.: Locally-adaptive and memetic evolutionary pattern search algorithms. Evol. Comput. 11, 29-52 (2003)

17. Hedar, A.-R., Fukushima, M.: Heuristic pattern search and its hybridization with simulated annealing for nonlinear global optimization. Optim. Methods Softw. 19, 291-308 (2004)

18. Hough, P., Kolda, T.G., Torczon, V.: Asynchronous parallel pattern search for nonlinear optimization. SIAM J. Sci. Comput. 23, 134-156 (2001)

19. Huyer, W., Neumaier, A.: Global optimization by multilevel coordinate search. J. Global Optim. 14, 331-355 (1999) http://solon.cma.univie.ac.at/ neum/software/mcs

20. Ingber, L.: Adaptative simulated annealing (ASA): lessons learned. Control Cybern. 25, 33-54 (1996) http://www.ingber.com

21. Ingber, L., Rosen, B.: Genetic algorithms and very fast simulated reannealing: a comparison. Math. Comput. Model. 16, 87-100 (1992)

22. James, F.: A review of pseudorandom number generators. Comput. Phys. Commun. 60, 329-344 (1990)

23. Jones, D.R., Perttunen, C.D., Stuckman, B.E.: Lipschitzian optimization without the Lipschitz constant. J. Optim. Theory Appl. 79, 157-181 (1993)

24. Kennedy, J., Eberhart, R.: Particle swarm optimization. In: Proceedings of the 1995 IEEE International Conference on Neural Networks, pp. 1942-1948, Perth, Australia. IEEE Service Center, Piscataway, NJ (1995)

25. Kiseleva, E., Stepanchuk, T.: On the efficiency of a global non-differentiable optimization algorithm based on the method of optimal set partitioning. J. Global Optim. 25, 209-235 (2003)

26. Kolda, T.G., Lewis, R.M., Torczon, V.: Optimization by direct search: new prespectives on some classical and modern methods. SIAM Rev. 45, 385-482 (2003)

27. Levine, D.: Users guide to the PGAPack parallel genetic algorithm library. Technical Report ANL-95/18, Argonne National Laboratory (1996) http://www.mcs.anl.gov/pgapack.html

28. Locatelli, M.: A note on the Griewank test function. J. Global Optim. 25, 169-174 (2003)

29. Locatelli, M., Schoen, F.: Fast global optimization of difficult Lennard-Jones clusters. Comput. Optim. Appl. 21, 55-70 (2002)

30. Marsden, A.L.: Aerodynamic noise control by optimal shape design. Ph.D thesis, Stanford University (2004)

31. Meza, J.C., Martinez, M.L.: On the use of direct search methods for the molecular conformation problem. J. Comput. Chem. 15, 627-632 (1994)

32. Mongeau, M., Karsenty, H., Rouzé, V., Hiriart-Urruty, J.-B.: Comparison of public-domain software for black box global optimization. Optim. Methods Softw. 13, 203-226 (2000)

33. Park, S.K., Miller, K.W.: Random number generators: good ones are hard to find. Commun. ACM 31, 1192-1201 (1988)

34. Parsopoulos, K.E., Plagianakos, V.P., Magoulas, G.D., Vrahatis, M.N.: Stretching technique for obtaining global minimizers through particle swarm optimization. In: Proceedings of the Particle Swarm Optimization Workshop, pp. 22-29, Indianapolis, USA (2001)

35. Schutte, J.F., Groenwold, A.A.: A study of global optimization using particle swarms. J. Global Optim. 31(1), 93-108 (2003) 